\title{
Database Fragmentation with Encryption: Under Which Semantic Constraints and A Priori Knowledge Can Two Keep a Secret?^
}

\author{
Joachim Biskup and Marcel Preuß \\ Technische Universität Dortmund, Dortmund, Germany \\ \{biskup, preuss\}@ls6.cs.tu-dortmund.de
}

\begin{abstract}
Database outsourcing to semi-honest servers raises concerns against the confidentiality of sensitive information. To hide such information, an existing approach splits data among two supposedly mutually isolated servers by means of fragmentation and encryption. This approach is modelled logic-orientedly and then proved to be confidentiality preserving, even if an attacker employs some restricted but nevertheless versatile class of a priori knowledge to draw inferences. Finally, a method to compute a secure fragmentation schema is developed.
\end{abstract}

Keywords: A Priori Knowledge, Confidentiality Constraint, Fragmentation, Inference-Proofness, Logic, Outsourcing, Semi-Honest Server.

\section{Introduction}

Database outsourcing faces two directly conflicting goals: it should both reduce storage and processing costs by storing data on external servers as well as provably comply with confidentiality requirements - in particular with privacy concerns - in spite of storing data externally [10]. A basic solution presented in [29] aims at resolving this conflict by means of the combined usage of fragmentation and encryption: a client's database relation is losslessly decomposed into (at least) two vertical fragments each of which is maintained by a different semihonest server; sensitive data is split into harmless parts, either by breaking an association or by separating an encrypted piece of data from the cryptographic key employed; moreover, the servers are (postulated to be) mutually isolated and each attacker is assumed to have access to at most one server.

Consequently, due to splitting, each attacker (identified with a server) only has accesses to non-sensitive data and, due to losslessness, an authorized user (identified with the client) can still reconstruct the original data while, due to isolation, only authorized users can do so.

Example 1. We consider the relational instance about medical data shown in the upper half of Fig.1. Suppose that social security numbers (SSN) should be hidden, as well as associations between a patient identified by his name (Name)

* This work has been supported by the DFG under grant SFB 876/A5.

L. Wang and B. Shafiq (Eds.): DBSec 2013, LNCS 7964, pp. 17-32 2013.

(C) IFIP International Federation for Information Processing 2013 


\begin{tabular}{l||l|l|l|l|l}
$R$ & SSN & Name & Illness & HurtBy & Doctor \\
\hline & 1234 & Hellmann & Borderline & Hellmann & White \\
& 2345 & Dooley & Laceration & McKinley & Warren \\
& 3456 & McKinley & Laceration & Dooley & Warren \\
& 3456 & McKinley & Concussion & Dooley & Warren
\end{tabular}

\begin{tabular}{|c|c|c|c|c|c|c|c|c|c|c|}
\hline$F_{1}$ & $\underline{\text { tid }}$ & SSN & Name & HurtBy & Doctor & $F_{2}$ & $\underline{\text { tid }}$ & SSN & HurtBy & Illness \\
\hline & 1 & $e_{S}^{1}$ & Hellmann & $e_{H}^{1}$ & White & & 1 & $\kappa_{S}^{1}$ & $\kappa_{H}^{1}$ & Borderline \\
\hline & 2 & $e_{S}^{2}$ & Dooley & $e_{H}^{2}$ & Warren & & 2 & $\kappa_{S}^{2}$ & $\kappa_{H}^{2}$ & Laceration \\
\hline & 3 & $e_{S}^{3}$ & McKinley & $e_{H}^{3}$ & Warren & & 3 & $\kappa_{S}^{3}$ & $\kappa_{H}^{3}$ & Laceration \\
\hline & 4 & $e_{S}^{4}$ & McKinley & $e_{H}^{\frac{1}{H}}$ & Warren & & 4 & $\kappa_{S}^{4}$ & $\kappa_{H}^{4}$ & Concussion \\
\hline
\end{tabular}

Fig. 1. A relational instance containing sensitive data items and associations together with a possible fragmentation with encryption

and an illness treated (Illness), between a patient (Name) and a person who caused an illness (HurtBy), and between an illness (Illness) and a person having caused that illness (HurtBy), respectively. The lower half of Fig. 1- exhibits a possible fragmentation with encryption: The sensitive association between Name and Illness is "broken" by separating the attribute Name in the fragment $F_{1}$ from the attribute Illness in the fragment $F_{2}$. The sensitive associations between Name and HurtBy and between Illness and HurtBy are made "invisible" by using encryption for the attribute HurtBy such that ciphertexts are stored in fragment $F_{1}$ and corresponding keys in fragment $F_{2}$. The sensitive attribute SSN is similarly treated by encryption. The newly introduced tuple identifiers (tid) ensure the losslessness of the vertical decomposition (see, e.g., [1]).

At first glance two semi-honest servers seem to "keep the secrets" declared in a confidentiality policy. However, a second thought raises some doubts on the actual achievements: though each server only stores data that is non-sensitive per se, an attacker might still be able to infer sensitive information by exploiting his a priori knowledge obtained from further sources. In particular, this a priori knowledge might comprise semantic constraints to be satisfied by the relation being decomposed and individual fact data stemming from the "outside world".

Example 2. Suppose an attacker has access to the fragment $F_{1}$ and knows a priori that Doctor White is a psychiatrist only treating patients suffering from the Borderline-syndrome. The attacker can then conclude that patient Hellmann suffers from the illness Borderline-syndrome, thereby violating the requirement that associations between a patient and an illness treated should be hidden. Moreover, if this attacker additionally knows that all patients suffering from the Borderline-syndrome have hurt themselves, the attacker can conclude that patient Hellmann has been hurt by Hellmann, thereby revealing an association between a patient and a person who caused an illness.

The first violation is enabled by a priori knowledge connecting a fact shown in the visible fragment with a fact in the hidden fragment, namely by means of the constant symbols White and Borderline. Similarly, the second violation is caused by a priori knowledge that connects two concepts across the decomposition, namely the concept of a patient and the concept of a hurt creator, where 
a concept will be formally represented by a variable ranging over the domain of an attribute. Such connections might "transfer information" between the visible fragment and the hidden fragment. In other words, an attacker a priori knowing such connections might infer hidden information from visible information. Next, we introduce a more abstract example in a more formal way.

Example 3. The client maintains a relational schema with relational symbol $R$, attribute set $A_{R}=\left\{a_{1}, a_{2}, a_{3}, a_{4}\right\}$ and the functional dependency $a_{2} \rightarrow a_{3}$ as a semantic constraint. Confidentiality interests are expressed by a set $\mathcal{C}=$ $\left\{\left\{a_{1}, a_{3}\right\},\left\{a_{4}\right\}\right\}$ of two confidentiality constraints: $\left\{a_{1}, a_{3}\right\}$ is intended to require to hide the associations between values of the attributes $a_{1}$ and $a_{3}$, and $\left\{a_{4}\right\}$ requires to hide single values of attribute $a_{4}$. The a priori knowledge comprises the functional dependency and a sentence expressing the following: "for some specific values $b$ and $c$ for the attributes $a_{2}$ and $a_{3}$, resp., there exist a value $X_{1}$ for attribute $a_{1}$ and a value $X_{4}$ for attribute $a_{4}$ such that the tuple $\left(a_{1}: X_{1}, a_{2}: b, a_{3}: c, a_{4}: X_{4}\right)$ is an element of the relational instance $r$ ". Furthermore, fragment $F_{1}$ has attribute set $A_{F_{1}}=\left\{t i d, a_{1}, a_{2}, a_{4}\right\}$ and fragment $F_{2}$ attribute set $A_{F_{2}}=\left\{t i d, a_{3}, a_{4}\right\}$ such that the common attribute $a_{4}$ is encrypted.

Let fragment $F_{1}$ exhibit a tuple (tid : no, $a_{1}: a, a_{2}: b, a_{4}:$ ran), where $n o$ is a tuple identifier and ran results from encryption. Combining the a priori knowledge with the tuple exhibited, an attacker might infer that the value $a$ for attribute $a_{1}$ is associated with the value $c$ for attribute $a_{3}$, thereby violating the confidentiality constraint $\left\{a_{1}, a_{3}\right\}$. Thus fragment $F_{1}$ is not inference-proof under the given assumptions. In contrast, fragment $F_{2}$ is harmless.

The a priori knowledge relates the fragments $F_{1}$ and $F_{2}$ by means of both the functional dependency using variables and the association fact about $a$ and $b$ dealing with constant symbols. Though taken alone, each of these items might be harmless, their combination turns out to be potentially harmful. The next example indicates that for the same underlying situation one fragmentation satisfying required confidentiality constraints might be better than another one.

Example 4. Modifying Example 3 such that $A_{F_{1}}=\left\{\right.$ tid, $\left.a_{1}, a_{4}\right\}$ and $A_{F_{2}}=$ $\left\{t i d, a_{2}, a_{3}, a_{4}\right\}$ would block the harmful inference. For, intuitively, the crucial fact about the association of $a$ with $b$ does not span across the decomposition.

More generally, we will investigate the following problems in this article:

- Given a fragmentation, identify conditions on the a priori knowledge to provably disable an attacker to infer sensitive information.

- Given some a priori knowledge, determine a fragmentation such that an attacker cannot infer sensitive information.

Our solutions will be based on a logic-oriented modelling of the fragmentation approach presented in 29] within the more general framework of Controlled Interaction Execution, CIE, as surveyed in [3. This framework assists a database owner in ensuring that each of his interaction partners can only obtain a dedicated inference-proof view on the owner's data: each of these views does not 
contain information to be kept confidential from the respective partner, even if this partner tries to employ inferences by using his a priori knowledge and his general awareness of the protection mechanism. Our main achievements can be summarized as follows and will be elaborated in the remainder as indicated:

- We formalize the fragmentation approach of [29] (Sect.2).

- We provide a logic-oriented modelling of that approach (Sect.3).

- We exhibit sufficient conditions to achieve confidentiality (Sect.4).

- We propose a method to compute a suitable fragmentation (Sect.55).

These results extend the previous work [5] in which a more simple approach to fragmentation proposed in [7] - splitting a relational instance into one externally stored part and one locally-held part without resorting to encryption - is formally analyzed to be inference-proof. In particular, the previous work is extended by a more detailed formal modelling of fragmentation including encryption of values, a more expressive class of sentences representing an attacker's a priori knowledge and a method to compute an inference-proof fragmentation.

\section{Confidentiality by Fragmentation}

In this section, we briefly formalize and extend the approach to fragmentation proposed in [29]. All data is represented within a single relational instance $r$ over a relational schema $\left\langle R\left|A_{R}\right| S C_{R}\right\rangle$ with relational symbol $R$ and the set $A_{R}=$ $\left\{a_{1}, \ldots, a_{n}\right\}$ of attributes, for simplicity assumed to have the same type given by the infinite set $\mathcal{U}$ of values. Moreover, the set $S C_{R}$ contains some semantic (database) constraints, which must be satisfied by the relational instance $r$.

The idea for achieving confidentiality basically lies in splitting the original instance $r$ vertically (i.e., by projections on subsets of $A_{R}$ ) into two fragment instances $f_{1}$ and $f_{2}$ each of which is stored on exactly one of the two external servers instead of $r$. Those confidentiality requirements which cannot be satisfied by just splitting instance $r$ are satisfied by encrypting the values of some attributes. Each "encrypted attribute" is contained in $f_{1}-$ storing ciphertexts as well as in $f_{2}$ - storing globally unique cryptographic keys.

We assume an encryption function Enc $: \mathcal{U} \times \mathcal{U} \rightarrow \mathcal{U}$ satisfying the group properties to achieve perfect (information-theoretic) security. A value of $\mathcal{U}$ might be used not only as a plaintext but also as a cryptographic key and a ciphertext. The decryption function is defined by $\operatorname{Dec}(e, \kappa)=v$ iff $\operatorname{Enc}(v, \kappa)=e$.

Definition 1 (Fragmentation). Given a relational schema $\left\langle R\left|A_{R}\right| S C_{R}\right\rangle$, a vertical fragmentation $(\mathcal{F}, \mathcal{E})$ of $\left\langle R\left|A_{R}\right| S C_{R}\right\rangle$ contains a set $\mathcal{E} \subseteq A_{R}$ of so-called "encrypted attributes" and a set $\mathcal{F}=\left\{\left\langle F_{1}\left|A_{F_{1}}\right| S C_{F_{1}}\right\rangle,\left\langle F_{2}\left|A_{F_{2}}\right| S C_{F_{2}}\right\rangle\right\}$ in which $\left\langle F_{1}\left|A_{F_{1}}\right| S C_{F_{1}}\right\rangle$ and $\left\langle F_{2}\left|A_{F_{2}}\right| S C_{F_{2}}\right\rangle$ are relational schemas called fragments of $(\mathcal{F}, \mathcal{E})$ both containing the distinguished attribute $a_{t i d} \notin A_{R}$ for tuple identifiers. Moreover, for $i \in\{1,2\}$, it holds that 


\begin{tabular}{l||c|c|c|c} 
& $A_{F_{i}} \backslash A_{R}$ & $\left(A_{F_{1}} \backslash \mathcal{E}\right) \cap A_{R}$ & $\mathcal{E} \cap A_{F_{i}} \cap A_{R}$ & $\left(A_{F_{2}} \backslash \mathcal{E}\right) \cap A_{R}$ \\
\hline$A_{R}$ & & $a_{1}, \ldots, a_{h}$ & $a_{h+1}, \ldots, a_{k}$ & $a_{k+1}, \ldots, a_{n}$ \\
$A_{F_{1}}$ & $a_{\mathrm{tid}}$ & $a_{1}, \ldots, a_{h}$ & $a_{h+1}, \ldots, a_{k}$ & \\
$A_{F_{2}}$ & $a_{\mathrm{tid}}$ & & $a_{h+1}, \ldots, a_{k}$ & $a_{k+1}, \ldots, a_{n}$
\end{tabular}

Fig. 2. Rearrangement of columns of $r, f_{1}$ and $f_{2}$

(i) $A_{F_{i}}:=\left\{a_{t i d}\right\} \cup \bar{A}_{F_{i}}$ with $\bar{A}_{F_{i}} \subseteq A_{R}$,

(ii) $S C_{F_{i}}:=\left\{a_{t i d} \rightarrow \bar{A}_{F_{i}}\right\}$ with $a_{t i d} \rightarrow \bar{A}_{F_{i}}$ being a functional dependency declaring $a_{t i d}$ as a primary key,

(iii) $\bar{A}_{F_{1}} \cup \bar{A}_{F_{2}}=A_{R}$ and $\bar{A}_{F_{1}} \cap \bar{A}_{F_{2}}=\mathcal{E}$.

Given a relational instance $r$ over $\left\langle R\left|A_{R}\right| S C_{R}\right\rangle$, the fragment instances $f_{1}$ and $f_{2}$ over $\left\langle F_{1}\left|A_{F_{1}}\right| S C_{F_{1}}\right\rangle$ and $\left\langle F_{2}\left|A_{F_{2}}\right| S C_{F_{2}}\right\rangle$ are created by inserting exactly both the tuples $\nu_{1}$ into $f_{1}$ and $\nu_{2}$ into $f_{2}$ for each tuple $\mu \in r$. Thereby,

(a) $\nu_{1}\left[a_{t i d}\right]=\nu_{2}\left[a_{t i d}\right]=v_{\mu}$ s.t. $v_{\mu}$ is a globally unique tuple identifier,

(b) $\nu_{i}[a]=\mu[a]$ for $i \in\{1,2\}$ and for each attribute $a \in\left(\bar{A}_{F_{i}} \backslash \mathcal{E}\right)$,

(c) $\nu_{1}[a]:=\operatorname{Enc}(\mu[a], \kappa)$ and $\nu_{2}[a]:=\kappa$ for each $a \in \mathcal{E}$ s.t. $\kappa$ is a cryptographic key being random but globally unique for each value of each tuple.

W.l.o.g. we suppose that $A_{R}:=\left\{a_{1}, \ldots, a_{h}, a_{h+1}, \ldots, a_{k}, a_{k+1}, \ldots, a_{n}\right\}$ is the set of attributes of $\left\langle R\left|A_{R}\right| S C_{R}\right\rangle$ and that the columns of the instances $r, f_{1}$ and $f_{2}$ are rearranged as visualized in Fig. 2. The columns $h+1, \ldots, k$ differ in the interpretation of the values stored in the instances $r, f_{1}$ and $f_{2}$ : although each of the tuples $\mu \in r, \nu_{1} \in f_{1}$ and $\nu_{2} \in f_{2}$ assign values to the attributes $a_{h+1}, \ldots, a_{k}$, $\mu\left[a_{j}\right]$ is a plaintext value, $\nu_{1}\left[a_{j}\right]$ is a ciphertext value and $\nu_{2}\left[a_{j}\right]$ is a cryptographic key. In contrast, for $a_{1}, \ldots, a_{h}\left(a_{k+1}, \ldots, a_{n}\right.$, respectively) corresponding tuples of $r$ and $f_{1}\left(r\right.$ and $\left.f_{2}\right)$ share the same combination of values.

To enable an authorized user having access to both fragment-instances $f_{1}$ and $f_{2}$ to query all information contained in the original instance $r$, fragmentation ensures that in $f_{1}$ and $f_{2}$ exactly those two tuples $\nu_{1} \in f_{1}$ and $\nu_{2} \in f_{2}$ corresponding to a tuple of $r$ share the same unique tuple ID (item (a) of Def.1). Thus, if $\nu_{1}\left[a_{\mathrm{tid}}\right]=\nu_{2}\left[a_{\mathrm{tid}}\right]$, two tuples $\nu_{1} \in f_{1}$ and $\nu_{2} \in f_{2}$ can be recomposed to a tuple of $r$ with the help of a binary operation denoted by $\diamond$.

As the goal is to achieve confidentiality by fragmentation, a formal declaration of confidentiality requirements is indispensable. In [2,9] this is obtained by defining a set of so-called confidentiality constraints on schema level.

Definition 2 (Confidentiality Constraint). A confidentiality constraint $c$ over a relational schema $\left\langle R\left|A_{R}\right| S C_{R}\right\rangle$ is a non-empty subset $c \subseteq A_{R}$.

Semantically, a confidentiality constraint $c$ claims that each combination of values allocated to the set $c \subseteq A_{R}$ of attributes in the original instance $r$ over schema $\left\langle R\left|A_{R}\right| S C_{R}\right\rangle$ should neither be contained completely in the unencrypted part of $f_{1}$ nor be contained completely in the unencrypted part of $f_{2}$.

Definition 3 (Confidentiality of Fragmentation). Let $\left\langle R\left|A_{R}\right| S C_{R}\right\rangle$ be a relational schema, $(\mathcal{F}, \mathcal{E})$ a fragmentation of $\left\langle R\left|A_{R}\right| S C_{R}\right\rangle$ according to Def. 1 
and $\mathcal{C}$ a set of confidentiality constraints over $\left\langle R\left|A_{R}\right| S C_{R}\right\rangle$ according to Def. Q $(\mathcal{F}, \mathcal{E})$ is confidential w.r.t. $\mathcal{C}$ iff $c \nsubseteq\left(A_{F_{1}} \backslash \mathcal{E}\right)$ and $c \nsubseteq\left(A_{F_{2}} \backslash \mathcal{E}\right)$ for each $c \in \mathcal{C}$.

Example 5. The fragmentation depicted in Fig.1 is confidential w.r.t. the set $\mathcal{C}=\left\{c_{1}, c_{2}, c_{3}, c_{4}\right\}$ of confidentiality constraints such that $c_{1}=\{\operatorname{SSN}\}, c_{2}=$ $\{$ Name, Illness $\}, c_{3}=\{$ Name, HurtBy $\}$, and $c_{4}=\{$ Illness, HurtBy $\}$.

\section{A Logic-Oriented View on Fragmentation}

In this section we will present a logic-oriented modelling of fragmentation, for conciseness mostly focussing on the attacker's point of view resulting from his knowledge of the fragment instance $f_{1}$, which is supposed to be known to him.

To set up the universe of discourse, we start by defining the set $\mathcal{P}$ of predicate symbols of a language $\mathscr{L}$ of first-order logic with equality. First, to model the attacker's knowledge about the fragment instance $f_{1}$, we need the predicate symbol $F_{1} \in \mathcal{P}$ with arity $k+1=\left|A_{F_{1}}\right|$ (including the additional tuple ID attribute plus $k$ original attributes (cf. Fig.2(2)). Second, to capture the attacker's awareness of the fragmentation, in particular his partial knowledge about the hidden original instance $r$ and the separated second fragmentation instance $f_{2}$, we additionally use the predicate symbols $R$ with arity $n=\left|A_{R}\right|$ and $F_{2}$ with arity $n-h+1=\left|A_{F_{2}}\right|$. Additionally, the distinguished predicate symbol $\equiv \notin \mathcal{P}$ is available in $\mathscr{L}$ for expressing equality.

We employ the binary function symbols $E$ and $D$ for modelling the attacker's knowledge about the encryption function Enc and the inverse decryption function Dec. Finally, we denote tuple values by elements of the set Dom of constant symbols, which will be employed as the universe of (Herbrand) interpretations for $\mathscr{L}$ as well. In compliance with CIE (e.g., [4]6]) this set is assumed to be fixed and infinite. Further, we have an infinite set Var of variables.

As usual, the formulas contained in $\mathscr{L}$ are constructed inductively using the quantifiers $\forall$ and $\exists$ and the connectives $\neg, \wedge, \vee$ and $\Rightarrow$. Closed formulas, i.e., formulas without free occurrences of variables, are called sentences. This syntactic specification is complemented with a semantics which reflects the characteristics of databases by means of so-called DB-Interpretations according to [46]:

Definition 4 (DB-Interpretation). Given the language $\mathscr{L}$ described above, an interpretation $\mathcal{I}$ over a universe $\mathcal{U}$ is a DB-Interpretation for $\mathscr{L}$ iff

(i) Universe $\mathcal{U}:=\mathcal{I}($ Dom $)=$ Dom,

(ii) $\mathcal{I}(v)=v \in \mathcal{U}$ for every constant symbol $v \in$ Dom,

(iii) $\mathcal{I}(E)(v, \kappa)=e$ iff $\operatorname{Enc}(v, \kappa)=e$, for all $v, \kappa, e \in \mathcal{U}$,

(iv) $\mathcal{I}(D)(e, \kappa)=v$ iff $\operatorname{Dec}(e, \kappa)=v$, for all $v, \kappa, e \in \mathcal{U}$,

(v) every $P \in \mathcal{P}$ with arity $m$ is interpreted by a finite relation $\mathcal{I}(P) \subset \mathcal{U}^{m}$,

(vi) the predicate symbol $\equiv \notin \mathcal{P}$ is interpreted by $\mathcal{I}(\equiv)=\{(v, v) \mid v \in \mathcal{U}\}$.

If item (U) is instantiated by taking the instances $r, f_{1}$ and $f_{2}$ as interpretations of $P=R, F_{1}$, and $F_{2}$, respectively, the resulting DB-Interpretation $\mathcal{I}_{r, f_{1}, f_{2}}$ - or just $\mathcal{I}_{r}$ for short if $f_{1}$ and $f_{2}$ are derived from $r$ according to Def.[1 - is called induced by $r$ (and $f_{1}$ and $f_{2}$ ). 
The notion of satisfaction/validity of formulas in $\mathscr{L}$ by a DB-Interpretation is the same as in usual first-order logic. A set $\mathcal{S} \subset \mathscr{L}$ of sentences implies / entails a sentence $\Phi \in \mathscr{L}$ (written as $\mathcal{S}=_{D B} \Phi$ ) iff each DB-Interpretation $\mathcal{I}$ satisfying $\mathcal{S}$ (written as $\mathcal{I} \models_{M} \mathcal{S}$ ) also satisfies $\Phi$ (written as $\left.\mathcal{I} \models_{M} \Phi\right)$.

Considering an attacker knowing the fragment instance $f_{1}$, the attacker's positive knowledge about the tuples explicitly recorded in $f_{1}$ can be simply modelled logic-orientedly by adding an atomic sentence $F_{1}\left(\nu\left[a_{\mathrm{tid}}\right], \nu\left[a_{1}\right], \ldots, \nu\left[a_{k}\right]\right)$ for each tuple $\nu \in f_{1}$. As the original instance $r$ - and so its fragment instance $f_{1}$ - is assumed to be complet 1 , each piece of information expressible in $\mathscr{L}$ which is not contained in $r$ ( $f_{1}$, resp.) is considered to be not valid by Closed World Assumption (CWA). The concept of DB-Interpretations fully complies with the semantics of complete relational instances. Accordingly, an attacker knows that each of the infinite combinations of values $\left(v_{\text {tid }}, v_{1}, \ldots, v_{k}\right) \in D o m^{k+1}$ not contained in any tuple of $f_{1}$ leads to a valid sentence $\neg F_{1}\left(v_{\text {tid }}, v_{1}, \ldots, v_{k}\right)$.

As this negative knowledge is not explicitly enumerable, it is expressed implicitly by a so-called completeness sentence (cf. [4]) having a universally quantified variable $X_{j}$ for each attribute $a_{j} \in A_{F_{1}}$ (sentence (2) of Def.5 below). This completeness sentence expresses that every constant combination $\left(v_{\text {tid }}, v_{1}, \ldots, v_{k}\right) \in$ $D o m^{k+1}$ (substituting the universally quantified variables $X_{\mathrm{tid}}, X_{1}, \ldots X_{k}$ ) either appears in $f_{1}$ or satisfies the sentence $\neg F_{1}\left(v_{\text {tid }}, v_{1}, \ldots, v_{k}\right)$. By construction, this completeness sentence is satisfied by any DB-Interpretation induced by $f_{1}$.

Example 6. For the medical example, the knowledge implicitly taken to be not valid by CWA can be expressed as the following completeness sentence:

$$
\begin{aligned}
& \left(\forall X_{t}\right)\left(\forall X_{S}\right)\left(\forall X_{N}\right)\left(\forall X_{H}\right)\left(\forall X_{D}\right)[ \\
& \left(X_{t} \equiv 1 \wedge X_{S} \equiv e_{S}^{1} \wedge X_{N} \equiv \text { Hellmann } \wedge X_{H} \equiv e_{H}^{1} \wedge X_{D} \equiv \text { White }\right) \vee \\
& \left(X_{t} \equiv 2 \wedge X_{S} \equiv e_{S}^{2} \wedge X_{N} \equiv \text { Dooley } \wedge X_{H} \equiv e_{H}^{2} \wedge X_{D} \equiv \text { Warren }\right) \vee \\
& \left(X_{t} \equiv 3 \wedge X_{S} \equiv e_{S}^{3} \wedge X_{N} \equiv \text { McKinley } \wedge X_{H} \equiv e_{H}^{3} \wedge X_{D} \equiv \text { Warren }\right) \vee \\
& \left(X_{t} \equiv 4 \wedge X_{S} \equiv e_{S}^{4} \wedge X_{N} \equiv \text { McKinley } \wedge X_{H} \equiv e_{H}^{4} \wedge X_{D} \equiv \text { Warren }\right) \vee \\
& \neg F_{1}\left(X_{t}, X_{S}, X_{N}, X_{H}, X_{D}\right)
\end{aligned}
$$

Based on the explanations given so far, an attacker's knowledge about the fragment instance $f_{1}$ can be formalized logic-orientedly as follows:

Definition 5 (Logic-Oriented View on $f_{1}$ ). Given a fragment instance $f_{1}$ over $\left\langle F_{1}\left|A_{F_{1}}\right| S C_{F_{1}}\right\rangle$ according to Def. 1 with $A_{F_{1}}=\left\{a_{t i d}, a_{1}, \ldots, a_{k}\right\}$, the positive knowledge contained in $f_{1}$ is modelled in $\mathscr{L}$ by the set of sentences

$$
d b_{f_{1}}^{+}:=\left\{F_{1}\left(\nu\left[a_{t i d}\right], \nu\left[a_{1}\right], \ldots, \nu\left[a_{k}\right]\right) \mid \nu \in f_{1}\right\} .
$$

The implicit negative knowledge contained in $f_{1}$ is modelled in $\mathscr{L}$ by the singleton set $d b_{f_{1}}^{-}$containing the completeness sentence

$$
\left(\forall X_{t i d}\right) \ldots\left(\forall X_{k}\right)\left[\bigvee_{\nu \in f_{1}}\left(\bigwedge_{a_{j} \in A_{F_{1}}}\left(X_{j} \equiv \nu\left[a_{j}\right]\right)\right) \vee \neg F_{1}\left(X_{t i d}, X_{1}, \ldots, X_{k}\right)\right] .
$$

1 Though not explicitly stated in [2]9, in this article we follow the usual intuitive semantics of complete instances. 
Moreover the functional dependency $a_{t i d} \rightarrow\left\{a_{1}, \ldots, a_{k}\right\} \in S C_{F_{1}}$ is modelled in $\mathscr{L}$ by the singleton set $f d_{F_{1}}$ containing the sentence

$$
\begin{array}{r}
\left(\forall X_{t i d}\right)\left(\forall X_{1}\right) \ldots\left(\forall X_{k}\right)\left(\forall X_{1}^{\prime}\right) \ldots\left(\forall X_{k}^{\prime}\right)\left[F_{1}\left(X_{t i d}, X_{1}, \ldots, X_{k}\right) \wedge\right. \\
\left.F_{1}\left(X_{t i d}, X_{1}^{\prime}, \ldots, X_{k}^{\prime}\right) \Rightarrow\left(X_{1} \equiv X_{1}^{\prime}\right) \wedge \ldots \wedge\left(X_{k} \equiv X_{k}^{\prime}\right)\right]
\end{array}
$$

Overall the logic-oriented view on $f_{1}$ in $\mathscr{L}$ is $d b_{f_{1}}:=d b_{f_{1}}^{+} \cup d b_{f_{1}}^{-} \cup f d_{F_{1}}$.

Proposition 1. Under the assumptions of Def.5, the sentences (11), (2) and (3) of $d b_{f_{1}}$ are satisfied by the DB-interpretation $\mathcal{I}_{r}$, i.e., $\mathcal{I}_{r} \models{ }_{M} d b_{f_{1}}$.

Proof. Direct consequence of the definitions.

An attacker is assumed to know the process of fragmentation as well as the schemas $\left\langle R\left|A_{R}\right| S C_{R}\right\rangle$ and $\left\langle F_{2}\left|A_{F_{2}}\right| S C_{F_{2}}\right\rangle$ of the instances kept hidden from him. Thus he can infer that for each tuple $\nu_{1} \in f_{1}$ there are tuples $\nu_{2} \in f_{2}$ and $\mu \in r$ satisfying the equation $\nu_{1} \diamond \nu_{2}=\mu$. So, an attacker knows all values assigned to the set $\left(A_{F_{1}} \backslash \mathcal{E}\right) \cap A_{R}$ of unencrypted attributes in $\mu$ from his knowledge of $\nu_{1}$, whereas in general he only knows the existence of values for the remaining attributes (sentence (4) of Def.6]below). Similarly, the attacker is not able to infer the cleartext values assigned to the attributes of $\mathcal{E}$ in $\mu$ : by the group properties of the encryption function, each ciphertext considered might be mapped to each possible cleartext without knowing the specific key hidden in fragment $f_{2}$.

Next, an attacker knows that a tuple $\nu_{2} \in f_{2}$ can only exist if also corresponding tuples $\nu_{1} \in f_{1}$ and $\mu \in r$ satisfying the equation $\nu_{1} \diamond \nu_{2}=\mu$ exist (sentence (5) of Def.6 below). According to the if-part of sentence (6) this requirement analogously holds for the existence of each tuple of $r$. The only-if-part of sentence (6) describes the fact that the (hypothetical) knowledge of both tuples $\nu_{1} \in f_{1}$ and $\nu_{2} \in f_{2}$ with $\nu_{1}\left[a_{\mathrm{tid}}\right]=\nu_{2}\left[a_{\mathrm{tid}}\right]$ would enable the attacker to reconstruct the tuple $\mu \in r$ satisfying $\mu=\nu_{1} \diamond \nu_{2}$ completely.

Based on the one-to-one correspondence between each tuple $\mu \in r$ and a tuple $\nu_{1} \in f_{1}\left(\nu_{2} \in f_{2}\right.$, resp.), observing that two different tuples $\nu_{1}, \nu_{1}^{\prime} \in f_{1}$ are equal w.r.t. the values allocated to the unencrypted attributes of $\left(A_{F_{1}} \backslash \mathcal{E}\right) \cap A_{R}$, an attacker can reason that there are also two tuples $\mu, \mu^{\prime} \in r$ which are equal w.r.t. the values allocated to these attributes, but differ in at least one of the values allocated to $A_{R} \backslash\left(A_{F_{1}} \backslash \mathcal{E}\right.$ ) (sentence (7) (sentence (8) in case of $f_{2}$ ) of Def.6 below). Otherwise, the instance $r$ would have duplicates.

Summarizing, and for now neglecting semantic constraints, an attacker's logicoriented view on the (hidden) instances $r$ and $f_{2}$ can be modelled as follows:

Definition 6 (Fragmentation Logic-Oriented). Let $(\mathcal{F}, \mathcal{E})$ be a fragmentation of a relational schema $\left\langle R\left|A_{R}\right| S C_{R}\right\rangle$ with instance $r$ and let $f_{1}$ and $f_{2}$ be the corresponding fragment instances over the fragments $\left\langle F_{1}\left|A_{F_{1}}\right| S C_{F_{1}}\right\rangle \in \mathcal{F}$ and $\left\langle F_{2}\left|A_{F_{2}}\right| S C_{F_{2}}\right\rangle \in \mathcal{F}$ according to Def. 1. 
The knowledge about $r$ and $f_{2}$ deduced from the knowledge of $f_{1}$ is expressed by

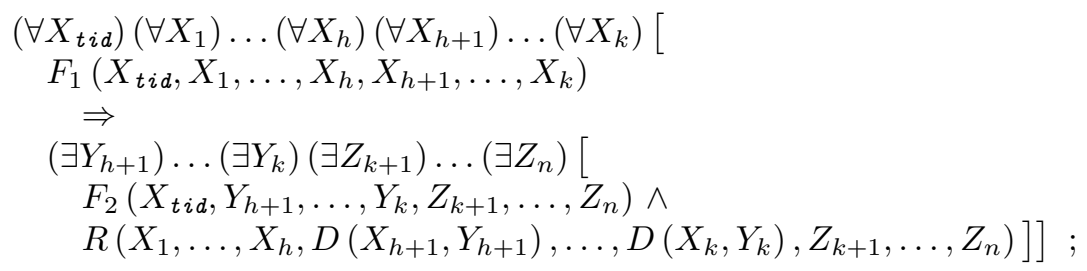

the knowledge about $r$ and $f_{1}$ deduced from the knowledge of $f_{2}$ is expressed by

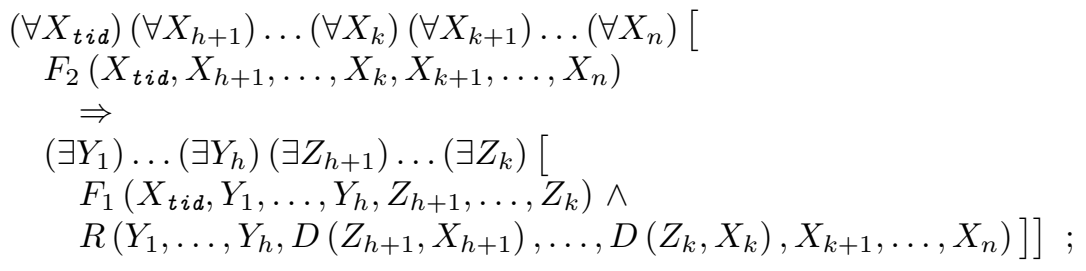

the knowledge about $f_{1}$ and $f_{2}$ deduced from the knowledge of $r$ as well as the knowledge about $r$ deduced from $f_{1}$ and $f_{2}$ is expressed by

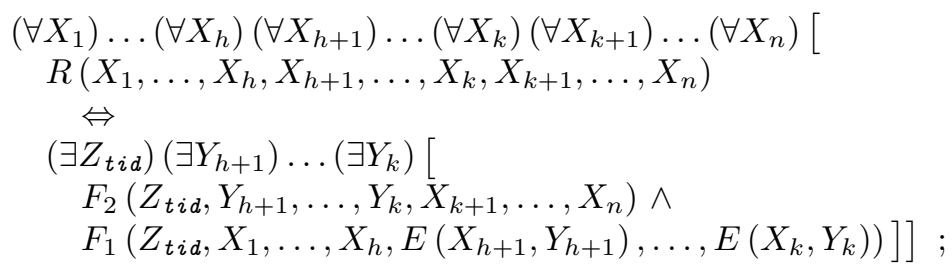

the knowledge about inequalities in $r$ based on $f_{1}$ is expressed by

$$
\begin{aligned}
& \left(\forall X_{t i d}\right)\left(\forall X_{t i d}^{\prime}\right)\left(\forall X_{1}\right) \ldots\left(\forall X_{h}\right)\left(\forall X_{h+1}\right) \ldots\left(\forall X_{k}\right)\left(\forall X_{h+1}^{\prime}\right) \ldots\left(\forall X_{k}^{\prime}\right)[ \\
& \quad\left[F_{1}\left(X_{t i d}, X_{1}, \ldots, X_{h}, X_{h+1}, \ldots, X_{k}\right) \wedge\right. \\
& \left.\quad F_{1}\left(X_{t i d}^{\prime}, X_{1}, \ldots, X_{h}, X_{h+1}^{\prime}, \ldots, X_{k}^{\prime}\right) \wedge\left(X_{t i d} \not \equiv X_{t i d}^{\prime}\right)\right] \\
& \quad(\exists \\
& \quad\left(\exists Y_{h+1}\right) \ldots\left(\exists Y_{n}\right)\left(\exists Z_{h+1}\right) \ldots\left(\exists Z_{n}\right)[ \\
& \quad R\left(X_{1}, \ldots, X_{h}, Y_{h+1}, \ldots, Y_{k}, Y_{k+1}, \ldots, Y_{n}\right) \wedge \\
& \left.\left.\quad R\left(X_{1}, \ldots, X_{h}, Z_{h+1}, \ldots, Z_{k}, Z_{k+1}, \ldots, Z_{n}\right) \wedge \bigvee_{j=h+1}^{n}\left(Y_{j} \not \equiv Z_{j}\right)\right]\right] ;
\end{aligned}
$$

and the knowledge about inequalities in $r$ based on $f_{2}$ is expressed by

$$
\begin{aligned}
& \left(\forall X_{t i d}\right)\left(\forall X_{t i d}^{\prime}\right)\left(\forall X_{h+1}\right) \ldots\left(\forall X_{k}\right)\left(\forall X_{h+1}^{\prime}\right) \ldots\left(\forall X_{k}^{\prime}\right)\left(\forall X_{k+1}\right) \ldots\left(\forall X_{n}\right)[ \\
& \quad\left[F_{2}\left(X_{t i d}, X_{h+1}, \ldots, X_{k}, X_{k+1}, \ldots, X_{n}\right) \wedge\right. \\
& \left.\quad F_{2}\left(X_{t i d}^{\prime}, X_{h+1}^{\prime}, \ldots, X_{k}^{\prime}, X_{k+1}, \ldots, X_{n}\right) \wedge\left(X_{t i d} \not \equiv X_{t i d}^{\prime}\right)\right] \\
& \quad\left(\exists Y_{1}\right) \ldots\left(\exists Y_{k}\right)\left(\exists Z_{1}\right) \ldots\left(\exists Z_{k}\right)[ \\
& \quad R\left(Y_{1}, \ldots, Y_{h}, Y_{h+1}, \ldots, Y_{k}, X_{k+1}, \ldots, X_{n}\right) \wedge \\
& \left.\left.\quad R\left(Z_{1}, \ldots, Z_{h}, Z_{h+1}, \ldots, Z_{k}, X_{k+1}, \ldots, X_{n}\right) \wedge \bigvee_{j=1}^{k}\left(Y_{j} \not \equiv Z_{j}\right)\right]\right]
\end{aligned}
$$

This view on $r$ and $f_{2}$ is referred to as the set of sentences $d b_{R}$ containing the sentences (4), (5), (6), (7) and (8). 
Strictly speaking, $d b_{R}$ alone does not provide any knowledge about the relational instance $r$; instead, only the combination of $d b_{f_{1}}$ and $d b_{R}$ describes the knowledge about $r$ that is available to an attacker. The essential part of this insight is formally captured by the following proposition.

Proposition 2. Under the assumptions of Def.[6, the sentences (4), (5), (6), (17) and (8) of $d b_{R}$ are satisfied by the DB-Interpretation $\mathcal{I}_{r}$, i.e., $\mathcal{I}_{r} \models_{M} d b_{R}$.

Proof. Omitted. See the informal explanations before Definition 6.

Note that - in contrast to sentence (6) - the equivalence does not hold for the sentences (4) and (5), as it can be shown by a straightforward example.

Finally, we have to model the confidentiality policy logic-orientedly. A confidentiality constraint $c \subseteq A_{R}$ claims that each combination of (cleartext-)values allocated to the attributes of $c$ should not be revealed to an attacker completely. To specify this semantics more precisely, it is assumed that $c$ only protects those combinations of values which are explicitly allocated to the attributes of $c$ in a tuple of $r$. In contrast, an attacker may get to know that a certain combination of values is not allocated to the attributes of $c$ in any tuple of $r$.

The wish to protect a certain combination of values $\left(v_{i_{1}}, \ldots, v_{i_{\ell}}\right) \in D_{o m}{ }^{|c|}$ is modelled as a "potential secret" in the form of a sentence $(\exists \boldsymbol{X}) R\left(t_{1}, \ldots, t_{n}\right)$ in which $t_{j}:=v_{j}$ holds for each $j \in\left\{i_{1}, \ldots, i_{\ell}\right\}$ and all other terms are existentially quantified variables. To protect each of the infinitely many combinations, regardless of whether it is contained in a tuple of $r$ or not, we use a single open formula with free variables $X_{i_{1}}, \ldots, X_{i_{\ell}}$ like an open query as follows.

Definition 7 (Confidentiality Policy). Let $\mathcal{C}$ be a set of confidentiality constraints over schema $\left\langle R\left|A_{R}\right| S C_{R}\right\rangle$ according to Def. D. Considering a confidentiality constraint $c_{i} \in \mathcal{C}$ with $c_{i}=\left\{a_{i_{1}}, \ldots, a_{i_{\ell}}\right\} \subseteq\left\{a_{1}, \ldots, a_{n}\right\}=A_{R}$ and the set $A_{R} \backslash c_{i}=\left\{a_{i_{\ell+1}}, \ldots, a_{i_{n}}\right\}$, constraint $c_{i}$ is modelled as a potential secret

$$
\Psi_{i}\left(\boldsymbol{X}_{\boldsymbol{i}}\right):=\left(\exists X_{i_{\ell+1}}\right) \ldots\left(\exists X_{i_{n}}\right) R\left(X_{1}, \ldots, X_{n}\right),
$$

which is a formula in the language $\mathscr{L}$. Thereby $\boldsymbol{X}_{\boldsymbol{i}}=\left(X_{i_{1}}, \ldots, X_{i_{\ell}}\right)$ is the vector of free variables contained in $\Psi_{i}\left(\boldsymbol{X}_{\boldsymbol{i}}\right)$. The set containing exactly one potential secret $\Psi_{i}\left(\boldsymbol{X}_{\boldsymbol{i}}\right)$ constructed as above for every confidentiality constraint $c_{i} \in \mathcal{C}$ is called potsec $(\mathcal{C})$. Moreover, the expansion $\operatorname{ex}(\operatorname{potsec}(\mathcal{C}))$ contains all ground substitutions over Dom of all formulas in $\operatorname{potsec}(\mathcal{C})$.

Example 7. For our example, $c_{2}=\{$ Name, Illness $\}$ is modelled as $\Psi_{2}\left(\boldsymbol{X}_{\mathbf{2}}\right):=$ $\left(\exists X_{S}\right)\left(\exists X_{H}\right)\left(\exists X_{D}\right) R\left(X_{S}, X_{N}, X_{I}, X_{H}, X_{D}\right)$ with free variables $\boldsymbol{X}_{\mathbf{2}}=\left(X_{N}, X_{I}\right)$.

\section{Inference-Proofness of Fragmentation}

Until now the logic-oriented modelling of an attacker's view only comprises knowledge the attacker can deduce from the outsourced fragment instance $f_{1}$, which is supposed to be visible to him. Additionally, however, the attacker might also employ a priori knowledge to draw harmful inferences. 
Example 8. As in Example 2, suppose the attacker knows that Doctor White is a psychiatrist only treating patients suffering from the Borderline-syndrome:

$\left(\forall X_{S}\right)\left(\forall X_{N}\right)\left(\forall X_{I}\right)\left(\forall X_{H}\right)\left[R\left(X_{S}, X_{N}, X_{I}, X_{H}\right.\right.$, White $) \Rightarrow\left(X_{I} \equiv\right.$ Borderline $\left.)\right]$.

This knowledge enables the attacker to conclude that patient Hellmann suffers from the illness Borderline-syndrome, thereby violating confidentiality constraint $c_{2}=\{$ Name, Illness $\}$. Moreover, let the attacker additionally know that all patients suffering from the Borderline-syndrome have hurt themselves:

$$
\left(\forall X_{S}\right)\left(\forall X_{N}\right)\left(\forall X_{H}\right)\left(\forall X_{D}\right)\left[R\left(X_{S}, X_{N} \text {, Borderline, } X_{H}, X_{D}\right) \Rightarrow\left(X_{N} \equiv X_{H}\right)\right] \text {. }
$$

The attacker can then draw the conclusion that patient Hellmann has been hurt by Hellmann, thereby violating $c_{3}=\{$ Name, HurtBy $\}$.

Following the framework of CIE [3, we aim at achieving a sophisticated kind of confidentiality taking care of an attacker's (postulated) a priori knowledge. This a priori knowledge is modelled as a finite set prior of sentences in $\mathscr{L}$ containing only $R$ and $\equiv$ as predicate symbols. Moreover, we always assume that the semantic constraints $S C_{R}$ declared in the relational schema are publicly known, i.e., $S C_{R} \subseteq$ prior. Intuitively, we then would like to guarantee that a fragmentation is inference-proof in the sense that - from the attacker's point of view - each of the potential secrets might not be true in the original relational instance $r$. More formally: for each potential secret $\Psi_{i}\left(\boldsymbol{v}_{\boldsymbol{i}}\right) \in \operatorname{ex}(\operatorname{potsec}(\mathcal{C}))$ there should exist an alternative instance $r^{\prime}$ over $\left\langle R\left|A_{R}\right| S C_{R}\right\rangle$ that witnesses the nonentailment $d b_{f_{1}} \cup d b_{R} \cup$ prior $\not \models_{D B} \Psi_{i}\left(\boldsymbol{v}_{\boldsymbol{i}}\right)$. Clearly, deciding on non-entailment, equivalently finding a suitable witness, is computationally infeasible in general. Accordingly, we will have to restrict on approximations and special cases.

Regarding approximations, we might straightforwardly require for the witness $r^{\prime}$ that for at least one $m \in\left\{i_{1}, \ldots, i_{\ell}\right\}$ the value $v_{m}$ appearing in the potential secret must not occur under the attribute $a_{m}$. Accordingly, we could try to substitute $v_{m}$ in the original instance $r$ by a newly selected constant symbol $v^{*}$ to obtain $r^{\prime}$. However, we also have to preserve indistinguishability of $r$ and $r^{\prime}$ by the attacker, and thus $m$ has to be chosen such that $a_{m} \notin\left(A_{F_{1}} \backslash \mathcal{E}\right)$. Furthermore, to fully achieve indistinguishability, the alternative instance $r^{\prime}$ has to coincide with the original instance $r$ on the part visible in fragment $f_{1}$, i.e., $\mathcal{I}_{r^{\prime}}==_{M} d b_{f_{1}}$, and modifying the original instance $r$ into the alternative $r^{\prime}$ should preserve satisfaction of the a priori knowledge, i.e., $\mathcal{I}_{r^{\prime}} \models_{M}$ prior.

Regarding special cases, we will adapt two useful properties known from relational database theory [1. Genericity of a sentence in $\mathscr{L}$ perceives constant symbols as being atomic and uninterpreted. Intuitively, all knowledge about a constant symbol arises from its occurrences in the relational instance $r$. Clearly, sentences with "essential" occurrences of constant symbols will not be generic. But in general "essential" occurrences of constant symbols are difficult to identify. Moreover, renaming $v_{m}$ by $v^{*}$ should not modify the fragment $f_{1}$ that is visible to the attacker. Typedness restricts the occurrences of a variable within a sentence to a single attribute (column), and thus prevents a "transfer of information" from a visible attribute to a hidden one. 
We will now state our main result about the achievements of fragmentation with encryption regarding preservation of confidentiality against an attacker who only has access to one of the fragment instances, here exemplarily to fragment instance $f_{1}$. Facing the challenges discussed above, this main result exhibits a sufficient condition for confidentiality. An inference-proof fragmentation of the running example in terms of Theorem 11 is presented in Example 9 of Sect.5]

Theorem 1 (Inference-Proofness on Schema Level). Let $\left\langle R\left|A_{R}\right| S C_{R}\right\rangle$ be a relational schema with $A_{R}=\left\{a_{1}, \ldots, a_{n}\right\}$ and $(\mathcal{F}, \mathcal{E})$ be a fragmentation with fragment $\left\langle F_{1}\left|A_{F_{1}}\right| S C_{F_{1}}\right\rangle \in \mathcal{F}$ that is confidential w.r.t. a set $\mathcal{C}$ of confidentiality constraints. Moreover, let $S C_{R} \subseteq$ prior be a set of sentences in $\mathscr{L}$ containing only $R$ and $\equiv$ as predicate symbols, satisfying the following restrictions:

- Untyped dependencies with constants: each $\Gamma \in$ prior is in the syntactic form of $(\forall \boldsymbol{x})(\exists \boldsymbol{y})\left[\bigvee_{j=1, \ldots, p} \neg A_{j} \vee A_{p+1}\right]$ with $A_{l}$ being an atom of the form $R\left(t_{l, 1}, \ldots, t_{l, n}\right)$ or $\left(t_{p+1,1} \equiv t_{p+1,2}\right)$ and $t_{j, i}$ is a variable or a constant symbol; moreover, w.l.o.g., equality predicates may only occur positively, and there might also be a conjunction of positively occurring $R$-atoms.

- Satisfiability: prior is DB-satisfiable and each $\Gamma \in$ prior is not DB-tautologic (and thus: each $\Gamma \in$ prior is range-restricted and does not contain an existentially quantified variable in the negated atoms (premises)).

- Compatibility with $(\mathcal{F}, \mathcal{E})$ and $\mathcal{C}$ : there is a subset $M \subseteq\{h+1, \ldots, n\}$ s.t.

(1) $M \cap\left\{i_{1}, \ldots, i_{\ell}\right\} \neq \emptyset$ for each $c_{i} \in \mathcal{C}$ with $c_{i}=\left(a_{i_{1}}, \ldots, a_{i_{\ell}}\right)$;

(2) for each $\Gamma \in$ prior there exists a partitioning $\mathcal{X}_{1}^{\Gamma} \cup \mathcal{X}_{2}^{\Gamma}=$ Var s.t.

(i) for each atom $R\left(t_{1}, \ldots, t_{n}\right)$ of $\Gamma$

- for all $j \in\{1, \ldots, n\} \backslash M$ term $t_{j}$ can either be a (quantified) variable of $\mathcal{X}_{1}^{\Gamma}$ or a constant symbol of Dom,

- for all $j \in M$ term $t_{j}$ must be a (quantified) variable of $\mathcal{X}_{2}^{\Gamma}$,

(ii) for each atom $\left(X_{i} \equiv X_{j}\right)$ of $\Gamma$ either $X_{i}, X_{j} \in \mathcal{X}_{1}^{\Gamma}$ or $X_{i}, X_{j} \in \mathcal{X}_{2}^{\Gamma}$,

(iii) for each atom $\left(X_{i} \equiv v\right)$ of $\Gamma$ with $v \in$ Dom variable $X_{i}$ is in $\mathcal{X}_{1}^{\Gamma}$.

Then, inference-proofness is achieved: For each instance $r$ over $\left\langle R\left|A_{R}\right| S C_{R}\right\rangle$ with fragment instance $f_{1}$ such that $\mathcal{I}_{r} \models_{M}$ prior and for each potential secret $\Psi_{i}\left(\boldsymbol{v}_{\boldsymbol{i}}\right) \in \operatorname{ex}(\operatorname{potsec}(\mathcal{C}))$ we have $d b_{f_{1}} \cup d b_{R} \cup$ prior $\not{ }_{D B} \Psi_{i}\left(\boldsymbol{v}_{\boldsymbol{i}}\right)$,

i.e., there exists an alternative instance $r^{\prime}$ over $\left\langle R\left|A_{R}\right| S C_{R}\right\rangle$ s.t.

(a) $\mathcal{I}_{r^{\prime}} \models{ }_{M} d b_{f_{1}} \cup d b_{R} \cup$ prior, and

(b) $\mathcal{I}_{r^{\prime}} \not \neq_{M} \Psi_{i}\left(\boldsymbol{v}_{\boldsymbol{i}}\right)$.

Proof (sketch). Consider any $\Psi_{i}\left(\boldsymbol{v}_{\boldsymbol{i}}\right) \in \operatorname{ex}(\operatorname{potsec}(\mathcal{C}))$ with $\boldsymbol{v}_{\boldsymbol{i}}=\left(v_{i_{1}}, \ldots, v_{i_{\ell}}\right)$. Then $c_{i}:=\left\{a_{i_{1}}, \ldots, a_{i_{\ell}}\right\} \in \mathcal{C}$, and thus by the assumptions there is an attribute $a_{m} \in c_{i}$ with $m \in M$; moreover, either $a_{m} \in \mathcal{E}$ or $a_{m} \in\left(\bar{A}_{F_{2}} \backslash \mathcal{E}\right)$.

Starting the construction of $r^{\prime}$ and thus of the induced $\mathcal{I}_{r^{\prime}}$, to ensure $\mathcal{I}_{r^{\prime}} \models M$ $d b_{f_{1}}$ according to Proposition 1, we define $f_{1}^{\prime}:=f_{1}$ and $\mathcal{I}_{r^{\prime}}\left(F_{1}\right):=f_{1}^{\prime}$.

Continuing the construction of $\mathcal{I}_{r^{\prime}}$, we select a constant symbol $v^{*} \neq v_{m}$ from the infinite set $\mathcal{U}$ that does not occur in the finite active domain of $\pi_{M}(r)$ and define a bijection $\varphi: \mathcal{U} \rightarrow \mathcal{U}$ such that $\varphi\left(v_{m}\right)=v^{*}$ and no value of $\pi_{M}(r)$ is mapped to $v_{m}$. Then we extend $\varphi$ to a tuple transformation $\varphi^{*}$ that maps a 
value $v$ for an attribute $a_{j} \in A_{R}$ with $j \in M$ to $\varphi(v)$ and each value for an attribute $a_{j} \in A_{R}$ with $j \notin M$ to itself, and define $r^{\prime}:=\varphi^{*}[r]$. Accordingly, the predicate symbol $R$ is interpreted by $\mathcal{I}_{r^{\prime}}(R):=r^{\prime}$.

The instance $r^{\prime}$ and its fragment instance $f_{1}^{\prime}$ together uniquely determine the corresponding fragment instance $f_{2}^{\prime}$ - whose constructability is guaranteed by the group properties of Enc - and thus we define $\mathcal{I}_{r^{\prime}}\left(F_{2}\right):=f_{2}^{\prime}$.

By the selection of $v^{*}$ and the definition of $\varphi$, we immediately have $\mathcal{I}_{r^{\prime}} \not \forall_{M}$ $\Psi_{i}\left(\boldsymbol{v}_{\boldsymbol{i}}\right)$, and thus $\mathcal{I}_{r^{\prime}}$ complies with property (b). Furthermore, by the construction and according to Proposition $2, \mathcal{I}_{r^{\prime}} \models_{M} d b_{R}$. Finally, we outline the argument to verify the remaining part of property (国), namely $\mathcal{I}_{r^{\prime}} \models_{M}$ prior.

We consider the following $\Gamma \in$ prior (other cases are treated similarly):

$$
(\forall \boldsymbol{x})(\exists \boldsymbol{y})\left[\bigvee_{j=1, \ldots, p} \neg R\left(t_{j, 1}, \ldots, t_{j, n}\right) \vee R\left(t_{p+1,1}, \ldots, t_{p+1, n}\right)\right],
$$

where $\left\{t_{j, 1}, \ldots, t_{j, n}\right\} \subseteq \boldsymbol{x} \cup \operatorname{Dom}$ for $j \in\{1, \ldots, p\}$ and $\left\{t_{p+1,1}, \ldots, t_{p+1, n}\right\} \subseteq$ $\boldsymbol{x} \cup \boldsymbol{y} \cup D o m$. To demonstrate $\mathcal{I}_{r^{\prime}} \equiv_{M} \Gamma$, we inspect any variable substitution $\sigma^{\prime}: \boldsymbol{x} \rightarrow$ Dom. If there exists $j \in\{1, \ldots, p\}$ such that $\mathcal{I}_{r^{\prime}}^{\sigma^{\prime}} \models_{M} \neg R\left(t_{j, 1}, \ldots, t_{j, n}\right)$, we are done.

Otherwise, for all $j \in\{1, \ldots, p\}$ we have $\mathcal{I}_{r^{\prime}}^{\sigma^{\prime}} \not \neq_{M} \neg R\left(t_{j, 1}, \ldots, t_{j, n}\right)$ and thus for each tuple $\mu_{j}^{\prime}:=\left(\sigma^{\prime}\left(t_{j, 1}\right), \ldots, \sigma^{\prime}\left(t_{j, n}\right)\right)$ we have $\mu_{j}^{\prime} \in r^{\prime}$. Since $r^{\prime}:=\varphi^{*}[r]$, for all $j \in\{1, \ldots, p\}$ there exists $\mu_{j} \in r$ such that $\varphi^{*}\left[\mu_{j}\right]=\mu_{j}^{\prime}$. Now exploiting the properties of the set $M$ - essentially, for each term exactly one case of the definition of $\varphi^{*}$ applies - we can construct a variable substitution $\sigma: \boldsymbol{x} \rightarrow$ Dom such that $\mu_{j}=\left(\sigma\left(t_{j, 1}\right), \ldots, \sigma\left(t_{j, n}\right)\right)$ and, accordingly, $\mathcal{I}_{r}^{\sigma} \not \nvdash_{M} \neg R\left(t_{j, 1}, \ldots, t_{j, n}\right)$.

Since $\mathcal{I}_{r} \models_{M} \Gamma$, there exists a variable substitution $\tau: \boldsymbol{y} \rightarrow$ Dom such that $\mathcal{I}_{r}^{\sigma \mid \tau}=_{M} R\left(t_{p+1,1}, \ldots, t_{p+1, n}\right)$, i.e., $\mu_{p+1}:=\left(\sigma\left|\tau\left(t_{p+1,1}\right), \ldots, \sigma\right| \tau\left(t_{p+1, n}\right)\right) \in r$. By the definition of $r^{\prime}$, we have $\mu_{p+1}^{\prime}:=\varphi^{*}\left[\mu_{p+1}\right] \in r^{\prime}$.

Exploiting the properties of $M$ and using $\tau$, we can construct a variable substitution $\tau^{\prime}: \boldsymbol{y} \rightarrow$ Dom such that $\mu_{p+1}^{\prime}=\left(\sigma^{\prime}\left|\tau^{\prime}\left(t_{p+1,1}\right), \ldots, \sigma^{\prime}\right| \tau^{\prime}\left(t_{p+1, n}\right)\right)$. Hence, $\mathcal{I}_{r^{\prime}}^{\sigma^{\prime} \mid \tau^{\prime}} \models_{M} R\left(t_{p+1,1}, \ldots, t_{p+1, n}\right)$ and thus $\mathcal{I}_{r^{\prime}}^{\sigma^{\prime} \mid \tau^{\prime}} \models_{M} \Gamma$.

Theorem 1 provides a sufficient condition for inference-proofness on schema level, i.e., for each relational instance satisfying the a priori knowledge prior. In some situations, however, a security officer might aim at only achieving inferenceproofness of a fixed particular relational instance $r$. Such a situation could be captured by a corollary. Essentially, if we know $r$ and thus also $f_{1}$ in advance, we can inspect the usefulness of each implicational sentence $\Gamma \in$ prior of form $(\forall \boldsymbol{x})(\exists \boldsymbol{y})\left[\bigvee_{j=1, \ldots, p} \neg A_{j} \vee A_{p+1}\right]$ to derive harmful information for the specific situation. If $r$ already satisfies $(\forall \boldsymbol{x})\left[\bigvee_{j=1, \ldots, p} \neg A_{j}\right]$, then we can completely discard $\Gamma$ from the considerations. More generally, we could only consider the effects of $\Gamma$ for those variable substitutions $\sigma$ of $\boldsymbol{x}$ that make $\left[\bigvee_{j=1, \ldots, p} \neg A_{j}\right]$ false for $r$.

\section{Creation of an Appropriate Fragmentation}

If an attacker is supposed to have a priori knowledge, a fragmentation has to comply with this knowledge to guarantee inference-proofness in terms of Theorem 1 


\begin{tabular}{c||c|c|l|c|l}
$F_{1}$ & $\underline{\text { tid }}$ & SSN & Illness & HurtBy & Doctor \\
\hline \multirow{6}{*}{} & 1 & $e_{S}^{1}$ & Borderline & $e_{H}^{1}$ & White \\
& 2 & $e_{S}^{2}$ & Laceration & $e_{H}^{2}$ & Warren \\
& 3 & $e_{S}^{3}$ & Laceration & $e_{H}^{3}$ & Warren \\
& 4 & $e_{S}^{4}$ & Concussion & $e_{H}^{4}$ & Warren
\end{tabular}

\begin{tabular}{c||c|c|c|l}
$F_{2}$ & tid & SSN & HurtBy & Name \\
\hline & 1 & $\kappa_{S}^{1}$ & $\kappa_{H}^{1}$ & Hellmann \\
& 2 & $\kappa_{S}^{2}$ & $\kappa_{H}^{2}$ & Dooley \\
& 3 & $\kappa_{S}^{3}$ & $\kappa_{H}^{3}$ & McKinley \\
& 4 & $\kappa_{S}^{4}$ & $\kappa_{H}^{4}$ & McKinley
\end{tabular}

Fig. 3. Inference-proof fragmentation w.r.t. a priori knowledge of Example 8

Hence, an algorithm computing a fragmentation should not only determine an arbitrary fragmentation being confidential in terms of Def.3. The algorithm should rather consider all of these fragmentations and select one complying with the user's a priori knowledge (if such a fragmentation exists).

Example 9. Reconsidering the a priori knowledge presented in Example 8 this knowledge does not compromise confidentiality if the fragmentation known from Fig.11 is modified as depicted in Fig. 3. In terms of Theorem 1, for an attacker knowing $f_{1}$ the set $M$ can be chosen to contain the indices of SSN, HurtBy and Name and for both sentences $\Gamma_{1}$ and $\Gamma_{2}$ of Example 8 the set of variables can be partitioned s.t., for both $i \in\{1,2\}, X_{I}, X_{D} \in \mathcal{X}_{1}^{\Gamma_{i}}$ and $X_{S}, X_{N}, X_{H} \in \mathcal{X}_{2}^{\Gamma_{i}}$.

In the following, an Integer Linear Program (ILP) (see [11) computing a confidential fragmentation complying with an attacker's a priori knowledge is developed to solve this problem with the help of generic algorithms solving ILPs 2 As the optimization goal the set of "encrypted attributes" is chosen to be minimized to reduce the costs for processing queries over the fragmented database as proposed in [29]. Other optimization goals are conceivable, too.

Given the attribute set $A_{R}$ of an original schema $\left\langle R\left|A_{R}\right| S C_{R}\right\rangle$, a set $\mathcal{C}$ of confidentiality constraints and a set prior in terms of Theorem 1 the ILP presented in the following computes the attribute sets $\bar{A}_{F_{1}}$ and $\bar{A}_{F_{2}}$ as well as the set $\mathcal{E}$ of "encrypted attributes" of a fragmentation being confidential w.r.t to $\mathcal{C}$ and complying with prior. The ILP contains the following binary decision variables:

- A variable $a_{j}^{i}$, for both $i \in\{1,2\}$ and for each $a_{j} \in A_{R}$. If $a_{j}^{i}=1$, attribute $a_{j} \in A_{R}$ is in $\bar{A}_{F_{i}}$; if $a_{j}^{i}=0$, attribute $a_{j} \in A_{R}$ is not in $\bar{A}_{F_{i}}$.

- A variable $a_{j}^{e}$ for each $a_{j} \in A_{R}$. If $a_{j}^{e}=1$, attribute $a_{j} \in A_{R}$ is an "encrypted attribute"; if $a_{j}^{e}=0$, attribute $a_{j} \in A_{R}$ is a "cleartext attribute".

- A variable $m_{j}$ for each $a_{j} \in A_{R}$. If $m_{j}=1$, the index of attribute $a_{j}$ is in $M$; if $m_{j}=0$, the index of attribute $a_{j}$ is not in $M$.

- A variable $X^{\Gamma}$ for each variable $X$ contained in a sentence $\Gamma \in$ prior. If $X^{\Gamma}=1$, variable $X$ is in $\mathcal{X}_{1}^{\Gamma}$; if $X^{\Gamma}=0$, variable $X$ is in $\mathcal{X}_{2}^{\Gamma}$.

For each $\Gamma \in$ prior the set $\operatorname{Var}_{j}^{\Gamma}$ is assumed to contain $X^{\Gamma}$ if $\Gamma$ is built over an atom $R\left(t_{1}, \ldots, t_{n}\right)$ with $t_{j}$ being the variable $X$ (note that each variable might occur in different columns). Moreover, the set $\operatorname{const}(\Gamma)$ is assumed to contain the index $j$, if $\Gamma$ is built over an atom $R\left(t_{1}, \ldots, t_{n}\right)$ with $t_{j}$ being a constant. Then, the ILP computing an appropriate fragmentation is defined as follows:

${ }^{2}$ For our prototype implementation "lp_solve" turned out to be an appropriate and fast ILP solver (see http://lpsolve.sourceforge.net/). 
Minimize the number of "encrypted attributes", i.e., min: $\sum_{j=1}^{n} a_{j}^{e}$ s.t. the following constraints are fulfilled:

- "Cleartext attributes" in exactly one fragment, "encrypted ones" in both: $a_{j}^{1}+a_{j}^{2}=1+a_{j}^{e}$ for each $a_{j} \in A_{R}$

- For $i \in\{1,2\}$, fragment $\left\langle F_{i}\left|A_{F_{i}}\right| S C_{F_{i}}\right\rangle$ fulfills all confidentiality constraints: $\sum_{a_{j} \in c} a_{j}^{i} \leq|c|-1+\sum_{a_{j} \in c} a_{j}^{e}$ for each $c \in \mathcal{C}$ and each $i \in\{1,2\}$

- $M \subseteq\{h+1, \ldots, n\}$, i.e., $M$ is a subset of attributes in $A_{F_{2}}$ : $m_{j} \leq a_{j}^{2}$ for each $a_{j} \in A_{R}$

- $M$ overlaps with the indices of the attributes of each $c \in \mathcal{C}$ :

$\sum_{a_{j} \in c} m_{j} \geq 1$ for each $c \in \mathcal{C}$

- For each formula $\Gamma \in$ prior:

- In each $R\left(t_{1}, \ldots, t_{n}\right)$ of $\Gamma$ : for each $t_{j}$ being a constant with $j \notin M$ : $m_{j}=0$ for each $j \in \operatorname{const}(\Gamma)$

- Partitioning of variables into $\mathcal{X}_{1}^{\Gamma}$ and $\mathcal{X}_{2}^{\Gamma}$ : $X^{\Gamma}=1-m_{j}$ for $j \in\{1, \ldots, n\}$ with $\operatorname{Var}_{j}^{\Gamma} \neq \emptyset$ and each $X^{\Gamma} \in \operatorname{Var}_{j}^{\Gamma}$

- In each atom $\left(X_{i} \equiv X_{j}\right)$ : variables $X_{i}, X_{j}$ belong to the same partition: $X_{i}^{\Gamma}=X_{j}^{\Gamma}$ for each atom $\left(X_{i} \equiv X_{j}\right)$

- In each atom $(X \equiv v)$ : variable $X$ belongs to partition $\mathcal{X}_{1}^{\Gamma}$ : $X^{\Gamma}=1$ for each atom $(X \equiv v)$

- Each decision variable of this ILP is binary:

$0 \leq x \leq 1$ for each integer decision variable $x$ of this ILP

If the ILP solver outputs a feasible solution, an inference-proof fragmentation can be determined by constructing the sets $\bar{A}_{F_{1}}, \bar{A}_{F_{2}}$ and $\mathcal{E}$ of Def.11 according to the allocation of the corresponding decision variables of the ILP.

Note that availability requirements such as storing a particular subset of attributes within the same (or even a particular) fragment or keeping the values of a particular attribute as cleartext values can be simply modelled by adding appropriate constraints, i.e., (in-)equations, to the ILP.

\section{Conclusion and Future Work}

Motivated by the question, whether splitting of data vertically over two semihonest servers guarantees confidentiality, the fragmentation model introduced in [2] is formalized, then modelled logic-orientedly and subsequently analyzed w.r.t. its inference-proofness. This analysis considers an attacker employing his a priori knowledge to draw harmful inferences and provides a sufficient condition to decide whether a given combination of a fragmentation and a priori knowledge is inference-proof w.r.t. a given confidentiality policy. Additionally, a generic ILP formulation computing such an inference-proof fragmentation is developed.

As Theorem 1 only states a sufficient condition for inference-proofness, there might be a more relaxed, most desirably even necessary definition of a priori knowledge still guaranteeing inference-proofness. A full characterization of inference-proofness could also provide a basis for deciding on the existence of a secure fragmentation for a given setting. 
Theorem 1 might be also enhanced in the spirit of $k$-anonymity by a more sophisticated definition of confidentiality guaranteeing that an "invisible value" cannot be narrowed down to a set of possible values of a certain cardinality. A further analysis of confidentiality assuming that commonly used encryption functions such as AES or RSA (which do not satisfy the group properties) come into operation is desirable, too. Although a formal analysis based on probability theory and complexity theory is indispensable to guarantee profound statements, we expect these encryption functions to be "sufficiently secure" in practice.

In this article and previously in [5] each one of two existing approaches to achieve confidentiality by vertical fragmentation is analyzed. As a third approach - using an arbitrary number of fragments which are all supposed to be known to an attacker - is presented in [8], a formal analysis of this approach in the spirit of Theorem 1 might be another challenging task for future work.

\section{References}

1. Abiteboul, S., Hull, R., Vianu, V.: Foundations of Databases. Addison-Wesley, Reading (1995)

2. Aggarwal, G., Bawa, M., Ganesan, P., Garcia-Molina, H., Kenthapadi, K., Motwani, R., Srivastava, U., Thomas, D., Xu, Y.: Two can keep a secret: A distributed architecture for secure database services. In: CIDR 2005, pp. 186-199 (2005)

3. Biskup, J.: Inference-usability confinement by maintaining inference-proof views of an information system. International Journal of Computational Science and Engineering 7(1), 17-37 (2012)

4. Biskup, J., Bonatti, P.A.: Controlled query evaluation with open queries for a decidable relational submodel. Annals of Mathematics and Artificial Intelligence 50(1-2), 39-77 (2007)

5. Biskup, J., Preuß, M., Wiese, L.: On the Inference-Proofness of Database Fragmentation Satisfying Confidentiality Constraints. In: Lai, X., Zhou, J., Li, H. (eds.) ISC 2011. LNCS, vol. 7001, pp. 246-261. Springer, Heidelberg (2011)

6. Biskup, J., Wiese, L.: A sound and complete model-generation procedure for consistent and confidentiality-preserving databases. Theoretical Computer Science 412(31), 4044-4072 (2011)

7. Ciriani, V., De Capitani di Vimercati, S., Foresti, S., Jajodia, S., Paraboschi, S., Samarati, P.: Keep a few: Outsourcing data while maintaining confidentiality. In: Backes, M., Ning, P. (eds.) ESORICS 2009. LNCS, vol. 5789, pp. 440-455. Springer, Heidelberg (2009)

8. Ciriani, V., De Capitani di Vimercati, S., Foresti, S., Jajodia, S., Paraboschi, S., Samarati, P.: Combining fragmentation and encryption to protect privacy in data storage. ACM Transactions on Information and System Security 13(3) (2010)

9. Ganapathy, V., Thomas, D., Feder, T., Garcia-Molina, H., Motwani, R.: Distributing data for secure database services. Transactions on Data Privacy 5(1), 253-272 (2012)

10. Hacigümüs, H., Mehrotra, S., Iyer, B.R.: Providing database as a service. In: ICDE 2002, pp. 29-40. IEEE Computer Society, Los Alamitos (2002)

11. Korte, B., Vygen, J.: Combinatorial Optimization: Theory and Algorithms, 5th edn. Algorithms and Combinatorics. Springer, Heidelberg (2012) 